\title{
STM-Induced $\mathrm{SiO}_{2}$ Decomposition on $\mathrm{Si}(110)^{*}$
}

\author{
Masahiro Yano, ${ }^{\dagger}$ Yuki Uozumi, Satoshi Yasuda, and Hidehito Asaoka ${ }^{\ddagger}$ \\ Advanced Science Research Center, Japan Atomic Energy Agency, \\ 2-4, Shirakata, Tokai-mura, Naka-gun, Ibaraki 319-1195, Japan \\ Chie Tsukada, Hikaru Yoshida, and Akitaka Yoshigoe \\ Material Science Research Center, Japan Atomic Energy Agency, \\ 1-1-1, Kouto, Sayo-cho, Sayo-gun, Hyogo 679-5148, Japan \\ (Received 30 January 2018; Accepted 26 July 2018; Published 11 August 2018)
}

\begin{abstract}
Real-time scanning tunneling microscope (STM) measurements are performed during the thermal decomposition of an oxide layer on $\mathrm{Si}(110)$. Voids in which only oxide is removed are formed during the real-time measurements, unlike the thermal decomposition in which bulk $\mathrm{Si}$ is desorbed with oxide. Analysis of the STM images reveals that the measurement induces the decomposition of the oxide layer resulting from electron injection into the defect sites. The activation energy of thermal decomposition decreases by $0.4 \mathrm{eV}$ in the range of $700-780^{\circ} \mathrm{C}$. [DOI: 10.1380/ejssnt.2018.370]
\end{abstract}

Keywords: Scanning tunneling microscopy; Silicon oxides; Surface chemical reaction

\section{INTRODUCTION}

Formation and removal techniques of thin $\mathrm{SiO}_{2}$ layer on $\mathrm{Si}$ are essential for fabrication of metal-oxidesemiconductor (MOS) field-effect transistor (FET) devices because the layer plays as gate oxide. To further develop the miniaturization and integration of MOS FET devices, thickness of the gate oxide layer must be reduced, which requires further understanding of the thermal stability of the oxide. Oxide layer decomposition also plays an important role in the fabrication of substrates for epitaxial growth because the substrates of the devices require clean surfaces that are obtained via the thermal decomposition of ultrathin oxide layers [1]. Thus, understanding the thermal stability of the oxide is crucial for the fabrication of Si-based devices.

Many studies focusing on the thermal decomposition of the oxide layer have obtained insight into the thermal stability characteristics. In particular, a time evolution analysis of morphology during the thermal decomposition of the oxide layer facilitates better understanding of the thermal decomposition mechanism [2-13]. Several evaluations revealed that a "void", where the oxide layer is decomposed and the pure Si surface is exposed, is nucleated on the oxide layer during the initial decomposition stage [2-10]. The void is believed to grow due to $\mathrm{SiO}_{2}$ decomposition at the void periphery via a reaction: $\mathrm{SiO}_{2}+\mathrm{Si} \rightarrow 2 \mathrm{SiO} \uparrow[4-6]$. Si monomers diffusing from the bottom and sidewall of the void are consumed during the reaction [7]. However, further detailed dynamics of the decomposition have not been found because these studies did not observe the decomposition in real-time.

A few real-time microscopic investigations on the morphology and dynamics of thermal decomposition of the

\footnotetext{
* This paper was presented at the 8th International Symposium on Surface Science, Tsukuba International Congress Center, Tsukuba, Japan, October 22-26, 2017.

$\dagger$ Corresponding author: yano.masahiro@jaea.go.jp

‡ Corresponding author: asaoka.hidehito@jaea.go.jp
}

oxide layer have been reported [11-13]. Real-time low energy electron microscope (LEEM) observations revealed that the decomposition temperature, void density and void size during the decomposition are affected by the preparation method and thickness of the oxide layer [12]. Real-time evaluation using an atomic force microscope $(\mathrm{AFM})$ revealed the formation of dots in the void during the decomposition [13]. However, these studies were performed in the micrometer scale and the morphology and dynamics of thermal decomposition in the atomic-scale are not still clarified.

Real-time measurement using scanning tunneling microscope (STM) is appropriate for further understanding the time evolution of atomic-scale morphology and dynamics. For example, real-time STM study of the thermal decomposition revealed that voids are randomly nucleated on ultrathin oxide layer during annealing [11]. This indicates that the void nucleation is not depend on defects.

STM analysis is a powerful tool for obtaining atomicscale information; however, the interpretation of resultant images can be difficult because a high electron beam dosing and a high electric field are applied under the oxide layer during measurements, causing significant influence on the thermal decomposition. A previous study confirmed that an electron-beam (e-beam) of $20 \mathrm{eV}$ emitted from an STM tip set at $100 \mathrm{~nm}$ above the surface induces selective $\mathrm{SiO}_{2}$ decomposition [14] characterized by the following reaction: $2 \mathrm{SiO}_{2} \rightarrow \mathrm{O}_{2} \uparrow+2 \mathrm{SiO} \uparrow$ [15]

This study verifies the effect of STM-induced $\mathrm{SiO}_{2}$ decomposition reaction on the thermal decomposition of the oxide layer on $\mathrm{Si}(110)$. $\mathrm{Si}(110)$ is used as the substrate because it is a candidate for high-speed MOS FET with a high hole mobility $[16,17]$ and has potential as a novel platform with uniformly shaped Si nanodots on it [18]. Analysis of STM images reveals that the dense e-beam emitted from the STM tip induces $\mathrm{SiO}_{2}$ decomposition and consequently, the thermal decomposition process under the scanning region is affected. The STM-induced decomposition also reduces the activation energy of the $\mathrm{SiO}_{2}$ decomposition. 

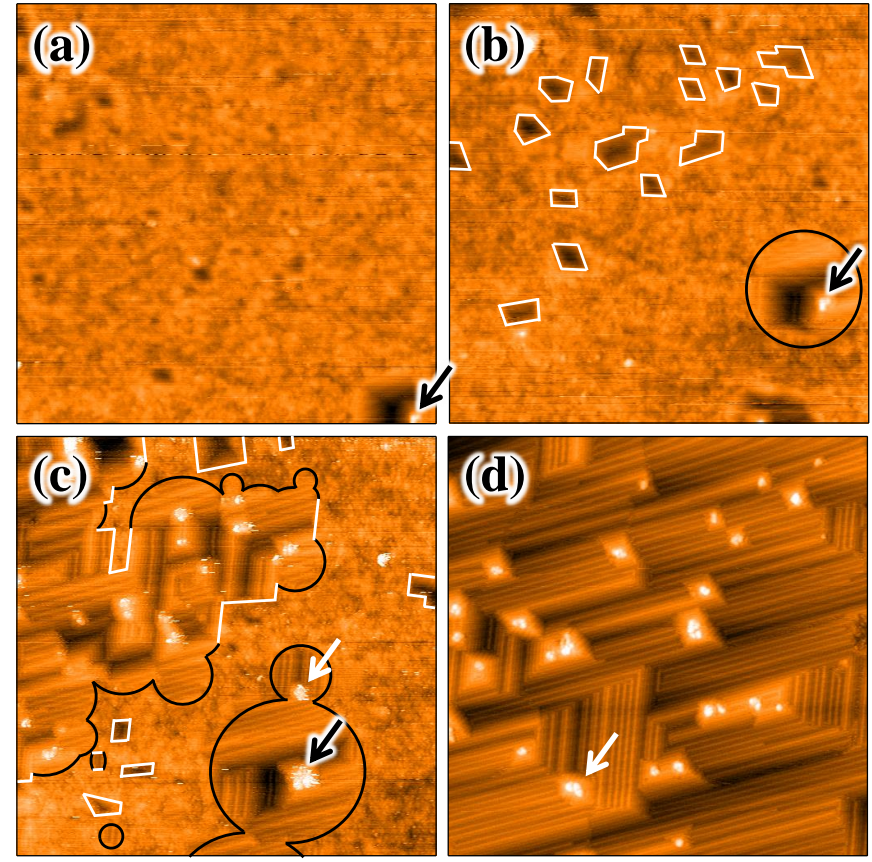

FIG. 1. STM images on the same scan area during the thermal decomposition of the oxide layer on $\operatorname{Si}(110)$ at $635^{\circ} \mathrm{C}$ for (a) 437-, (b) 704-, (c) 991-, and (d) 1753-min scanning durations $\left(V_{\mathrm{s}}=+7.0 \mathrm{~V}, I_{\mathrm{t}}=0.1 \mathrm{nA}, 300 \mathrm{~nm} \times 300 \mathrm{~nm}\right)$. The scans were performed every $4 \mathrm{~min}$. Black and white arrows denote same points, respectively. Black curves and white lines are position of shallow and deep void edges, respectively.

\section{EXPERIMENTAL}

A medium-doped n-type $\mathrm{Si}(110)$ substrate with a resistivity of $0.5-1.5 \Omega \mathrm{cm}$ was used. The $0.28-\mathrm{mm}$-thick wafer was cut into $1 \times 7 \mathrm{~mm}^{2}$ sections in the length direction, parallel to the $[1 \overline{1} 2]$ direction. Surface contamination removal and oxidation were performed using Shiraki's method [1]. This method uses hydrochloric acid to form the $5-8$-A-thick oxide layer [1]. We confirmed that the thickness of the oxide layer estimated via X-ray photo emission spectroscopy (XPS) was $5.8 \AA$. The thinness allowed electron tunneling, thereby implying that STM can measure the oxide surface. The sample was degassed at $550^{\circ} \mathrm{C}$ for $12 \mathrm{~h}$ in ultrahigh vacuum (UHV) before the decomposition of the oxide layer. The oxide layer was thermally decomposed, and the morphology and electronic state evolution during the decomposition were measured by STM (JEOL JSPM-4500A) and highly sensitive synchrotron radiation XPS, respectively. Oxidized Si surfaces required a sample bias voltage $>+6 \mathrm{~V}$ for STM measurements. The XPS experiments were conducted using SUREAC2000 at the BL23SU of Spring-8, Japan. An SR beam with a photon energy of $690 \mathrm{eV}$ calibrated by measuring the $\mathrm{Au}$ ff binding energy was used. The total energy resolution was $<0.25 \mathrm{eV}$, and the take-off angle of photoelectrons was $20^{\circ}$. These measurements were performed without taking samples out of vacuum. The sample was also annealed to nucleate voids (e.g., five days at $635^{\circ} \mathrm{C}$ or $28 \mathrm{~min}$ at $750^{\circ} \mathrm{C}$ ). The temperature was measured using radiation thermometer. The pressure in the UHV chamber was maintained $<3 \times 10^{-8}$ Pa during the decomposition.
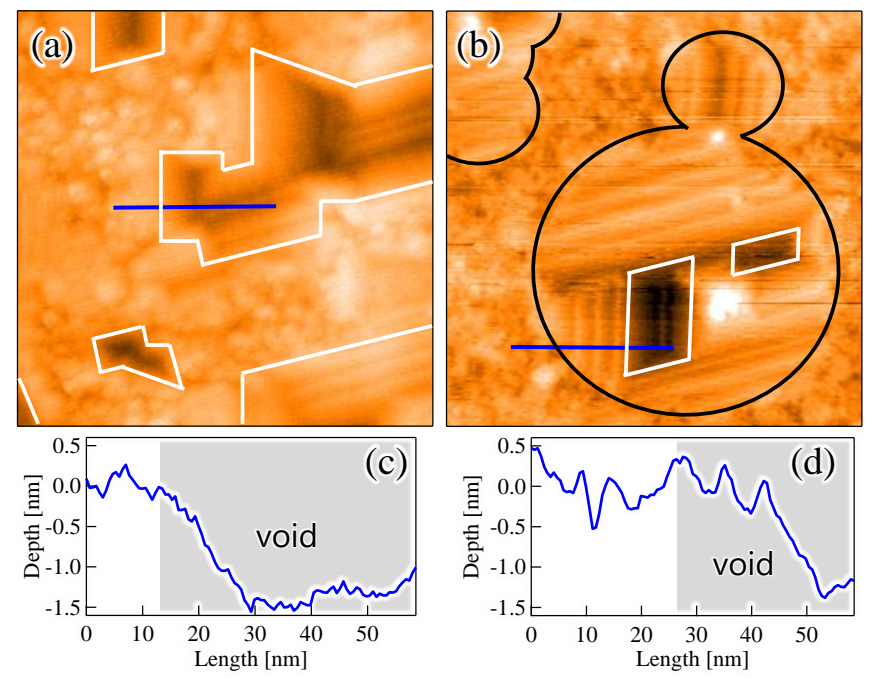

FIG. 2. STM images of oxide layer decomposed (a) a few centimeters away from STM tip and (b) during STM measurement on $\mathrm{Si}(110)$. STM measurements were performed following conditions; (a) $V_{\mathrm{s}}=+6.0 \mathrm{~V}, I_{\mathrm{t}}=0.03 \mathrm{nA}, 150 \mathrm{~nm} \times$ $150 \mathrm{~nm}$ and $(\mathrm{b}) V_{\mathrm{s}}=+7.0 \mathrm{~V}, I_{\mathrm{t}}=0.1 \mathrm{nA}, 150 \mathrm{~nm} \times 150 \mathrm{~nm}$. Black curves and white lines are position of shallow and deep void edges, respectively. (c) and (d) shows the height profiles of the blue lines in (a) and (b), respectively.

\section{RESULTS AND DISCUSSION}

Real-time STM measurement during the thermal decomposition of the oxide layer on $\mathrm{Si}(110)$ was performed to evaluate the STM-induced reaction's effect on thermal decomposition. Figure 1 shows the same scan area during the thermal decomposition of oxide layer on $\mathrm{Si}(110)$ at $635^{\circ} \mathrm{C}$. At the initial stage, several holes were formed due to silicon oxide desorption, indicating the nucleation of the void on the oxide layer [Fig. 1(a)]. The number and size of voids increased during annealing [Fig. 1(b)]. The voids were surrounded by an area that was different from the oxide surface. This area contained shallower voids than the initially formed voids because it had a stripedcontrast, indicating the $\mathrm{Si}(110)-16 \times 2$ reconstructed structure. The shallower voids grew isotropically and were round, as shown in Fig. 1(c). Furthermore, several dots, such as those pointed out by arrows in Fig. 1(c), were formed in the voids. Recently, we reported that the dots are continuous Si crystal from the bulk and are formed as uniformed shape on the reconstructed structure [18]. In the final stage, all voids coalesced with each other and the entire surface exhibited the reconstructed structure [Fig. 1(d)].

STM observation revealed the nanoscale morphology and dynamics during the decomposition. However, the STM-tip affects the decomposition. The shape and depth of voids affected and not affected by STM during growth were compared to clarify the STM-tip influences.

Figure 2(a) shows an STM image of oxide $\mathrm{Si}(110)$ recorded halfway through decomposition at $650^{\circ} \mathrm{C}$. The decomposition was performed several centimeters away from the STM tip; thus, the decomposition was not affected by STM. Voids had straight edges along the [112] and $[1 \overline{1} 2]$ directions, which were parallel to step rows of 


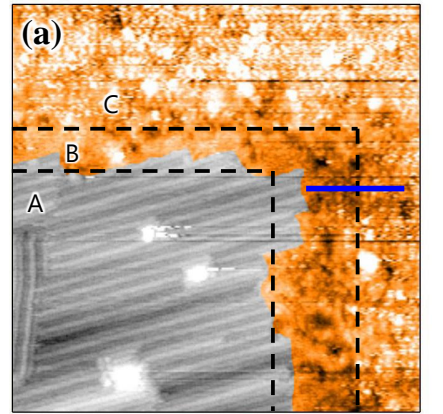

(b)

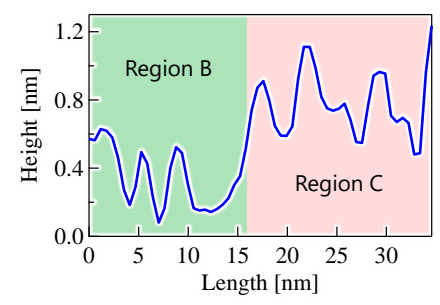

FIG. 3. (a) Enlarged STM image around edge of scanning area $\left(V_{\mathrm{s}}=+7.0 \mathrm{~V}, I_{\mathrm{t}}=0.1 \mathrm{nA}, 150 \mathrm{~nm} \times 150 \mathrm{~nm}\right)$. Two broken lines separate the image into three regions, as labeled with $\mathrm{A} / \mathrm{B} / \mathrm{C}$. The region $\mathrm{A}, \mathrm{B}$, and $\mathrm{C}$ were undergone $>300$, 25 , and 5 STM scans, respectively. The gray area denotes $16 \times 2$ reconstructed region. (b) The height profile of the blue line in (a).

the $16 \times 2$ reconstructed structure. Each void had a single domain of reconstructed structure, excepting the case that multiple voids coalesced. This indicated that the reconstruction did not multinucleate in each void because the initially formed reconstruction domain grew with the expansion of the void bottom.

By contrast, voids formed during STM measurements were round as shown in Fig. 2(b). Moreover, several voids had multiple reconstruction domains. Multiple nucleation of the reconstruction domain occurred between the void edge and the previously formed reconstruction domain. This suggested that the growth rate of the void was faster than that of the reconstruction domain.

The difference is also found in the void depth. Figures $2(\mathrm{c})$ and $2(\mathrm{~d})$ show the height profiles corresponding to the blue lines in Figs. 2(a) and 2(b), respectively. The void, which was not affected by STM measurements, was a deep hole because the bulk Si atoms in the substrate were consumed in the reaction [Fig. 2(c)]. In contrast, the void formed during STM measurements had a shallow area [Fig. 2(d)], which was formed by the decomposition of $\mathrm{SiO}_{2}$ without consuming bulk Si. Therefore, the STMtip decomposed the oxide layer via a process different from thermal decomposition and grew voids faster.

The features of the holes in the initial stage of decomposition [white quadrangle in Fig. 2(b)] were similar to those of voids that were unaffected by STM measurements [Fig. 2(a)]. The edges were along the [112] or [11ㄹ] directions, and the depths were $\sim 1.5 \mathrm{~nm}$. Thus, the initial void formation was the thermal decomposition of $\mathrm{SiO}_{2}$ consuming bulk Si even when affected by STM.

We have confirmed the difference in the decomposition process near the void edge by comparing different void shapes. The results indicate that the STM decomposed the oxide layer. The change in the height of the oxide surface versus the number of STM scans was analyzed to clarify influence of decomposition by the STM on oxide layer thickness.

Figure 3(a) is STM image which displays three regions undergone different numbers of STM scans, as labeled with $\mathrm{A} / \mathrm{B} / \mathrm{C}$. The $16 \times 2$ reconstructed structure was formed in the region $\mathrm{A}$ where was undergone $>300$ scans indicating the oxide layer was completely removed. The blue line transverses the region $\mathrm{B}$ (25 scans) and $\mathrm{C}$ (5 scans) and indicates the position of the height profile, as shown in Fig. 3(b). The height decreased as the number of scans increased, indicating that the STM measurement etched the oxide layer. This suggests that the STM decomposes the oxide layer and make it thin. This finally completely removes oxide resulting that pure $\mathrm{Si}$ surface is exposed such as the region A. In addition, the edge of oxide layer is easier to decompose than the terrace because there is always next to defect. This makes shallow part around deep void.

A possible cause of the decomposition of the oxide layer is the tunnel current from the STM-tip. A previous study reported that an e-beam of $20 \mathrm{eV}$ decomposed the thermal oxide layer [14]. The $\mathrm{SiO}_{2}$ decomposition by the e-beam is believed to follow the reaction $2 \mathrm{SiO}_{2} \rightarrow \mathrm{O}_{2} \uparrow+2 \mathrm{SiO} \uparrow$ [15] and does not consume bulk $\mathrm{Si}$ atoms to reduce $\mathrm{SiO}_{2}$. Furthermore, the extreme condition of STM observation (bias voltage $V_{\mathrm{s}}=+10 \mathrm{~V}$, tunnel current $I_{\mathrm{t}}=6 \mathrm{nA}$ ) created a leakage site of current on the thermal oxide layer [20]. For this phenomenon to occur, it is believed that the injection of electrons into a defect, which acts as an electron trap site, is essential [20]. The bond energy of $\mathrm{Si}-\mathrm{O}$ in $\mathrm{SiO}_{2}$ is $6.5 \mathrm{eV}$; therefore, the current injection from the STM-tip can cut the $\mathrm{Si}-\mathrm{O}$ bond. However, the STM could not decompose the thermally oxidized surface [20]. The difference should be caused by the difference of defect density. The oxide used in this study was formed via Shiraki's method. This forms oxide layer containing many defects. The defects make the oxide layer easier to decomposed than thermal oxide layer $[1,21]$. Furthermore, the sample was annealed for a long time period before STM measurement to nucleate voids. This should also make starting point of decomposition by the STM.

Although the electric field between the surface and the STM-tip was very high, field evaporation would not cause of decomposition of oxide layer. The electronic field between the surface and the tip was $\sim 7 \times 10^{9} \mathrm{~V} / \mathrm{m}$ because the distance and the sample bias voltage were $\sim 1 \mathrm{~nm}$ and $V_{\mathrm{s}}=7 \mathrm{~V}$, respectively. The electronic field was almost identical to the condition of typical $\mathrm{W}$ tip field evaporation, which was reported to be $4 \times 10^{10} \mathrm{~V} / \mathrm{m}$ via field ion microscope [19]. However, since the electric field does not affect the decomposition of the oxide layer [20], the electric field is not the mechanism of decomposition.

The STM-tip did not mechanically etch the oxide surface. Although the etching proceeded in the scan direction, the void grew isotropically. Furthermore, mechanical etching would deposit shavings at the end of scan but such a deposition was not found in Fig. 3(a).

The activation energy of oxide layer decomposition affected by STM measurement was compared with that of purely thermal decomposition of oxide layer estimated using XPS to quantitatively evaluate the effect of STM. The analysis was performed at a temperature $>700^{\circ} \mathrm{C}$. The reconstructed structure cannot exist at this temperature because phase transition to 11 structure occurs at $695^{\circ} \mathrm{C}$ [22]. Thus, obtained activation energy is not affected by formation of reconstructed structure.

The activation energy with less influence other than thermal decomposition was estimated via XPS because the electrons traveling from the oxide to the cathode cannot form a leakage site [20]. The activation energy was 

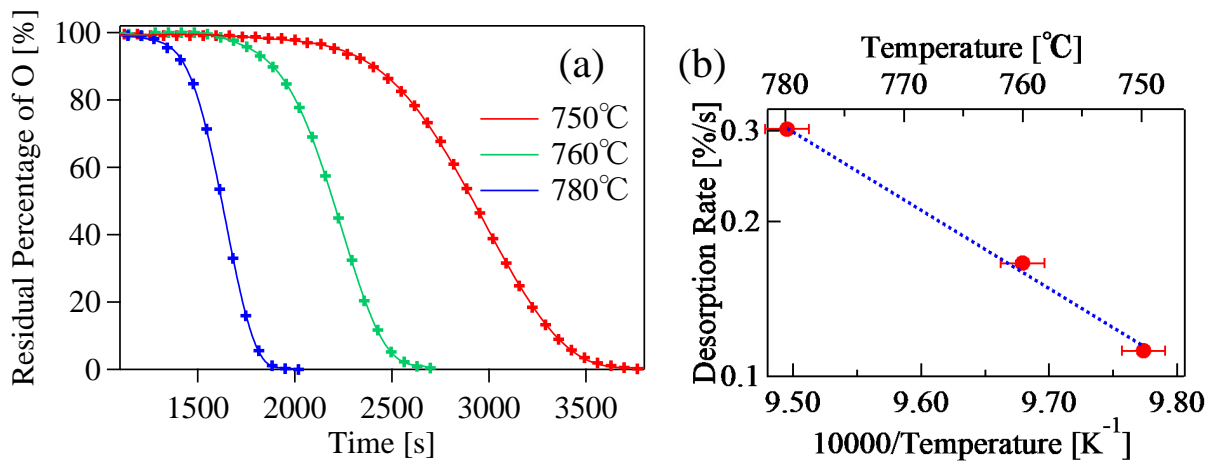

FIG. 4. (a) The time evolution of residual oxide during oxide layer decomposition on $\operatorname{Si}(110)$. (b) The Arrhenius plot of thermal decomposition rates estimated at $750^{\circ} \mathrm{C}, 760^{\circ} \mathrm{C}$, and $780^{\circ} \mathrm{C}$ as a function of $10000 / T$.

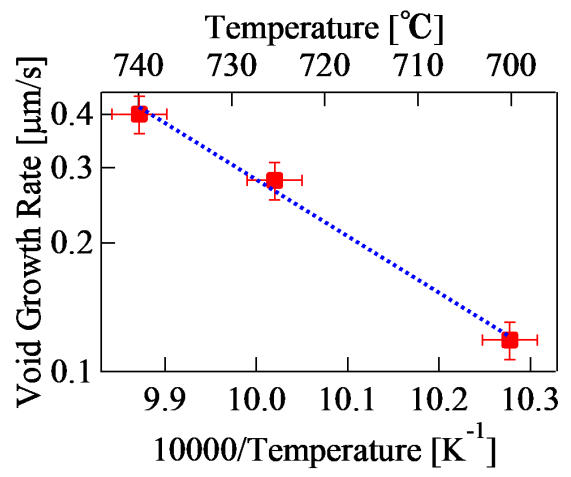

FIG. 5. Arrhenius plot of void growth rates estimated at $700^{\circ} \mathrm{C}, 735^{\circ} \mathrm{C}$, and $740^{\circ} \mathrm{C}$ as a function of $10000 / T$.

determined from the reduction rates of oxygen estimated from O 1s spectra measured via XPS. The amount of oxygen remained constant (for example, $28 \mathrm{~min}$ at $750^{\circ} \mathrm{C}$ ) after annealing began. Figure 4(a) shows the residuals of oxygen with respect to the initial amount. The beginning of the changing oxygen amount indicated the nucleation of a void. Since the number of voids increased, the reduction rate of the residual accelerated during the first half of the decomposition. However, in the second half of the decomposition, the reduction rate decelerated because the void edge wherein the decomposition reaction occurred was shortened by combining voids. Therefore, the maximum rate, where the acceleration and the deceleration were balanced, was adopted to determine the activation energy.

Figure 4(b) shows the Arrhenius plot of thermal decomposition rates estimated at $750^{\circ} \mathrm{C}, 760^{\circ} \mathrm{C}$, and $780^{\circ} \mathrm{C}$ as a function of $10000 / T$. The solid line indicates the result of fitting the date to the Arrhenius form, $R=$ $R_{0} \exp \left(-E_{\mathrm{a}} / k_{\mathrm{B}} T\right)$, where $R$ is the decomposition rate and $E_{\mathrm{a}}$ is the activation energy. The activation energy obtained from the plots was $3.0 \mathrm{eV}$.

This activation energy is $1.2 \mathrm{eV}$ lower than that of the thermal oxide layer on $\mathrm{Si}(100)$ determined via LEEM observation [12] possibly because of the oxidation method and surface orientation of the Si substrate. The oxide formed using hydrochloric acid used herein included several defects derived from $\mathrm{Cl}$ inclusion, leading to low temperature decomposition compared to thermal oxide $[1,21]$. The $\mathrm{Si}(110)$ surface was rapidly oxidized in an oxygen atmosphere, indicating the low activation energy of the $\mathrm{Si}+\mathrm{O}_{2} \rightarrow \mathrm{SiO}_{2}$ reaction on the $\mathrm{Si}(110)$ surface $[23,24]$. Since the free energy of $\mathrm{Si}, \mathrm{O}_{2}$, and $\mathrm{SiO}_{2}$ do not depend on surface orientation, the activation energy of decomposition should be also low.

Figure 5 shows the Arrhenius plot of void growth rate evaluated from STM observation at $700^{\circ} \mathrm{C}, 725^{\circ} \mathrm{C}$, and $740^{\circ} \mathrm{C}$ as a function of $10000 / T$ with a $+6 \mathrm{~V}$ sample bias voltage. The solid line indicates the result of fitting the date to the Arrhenius form, $r=r_{0} \exp \left(-E_{\mathrm{a}} / k_{\mathrm{B}} T\right)$, where $r$ is the differentiation of void diameter. The activation energy obtained from the plots was $2.6 \mathrm{eV}$, which was $0.4 \mathrm{eV}$ lower than that determined via XPS measurements. Therefore, STM measurements reduce the activation energy of oxide layer decomposition on $\mathrm{Si}(110)$ by $0.4 \mathrm{eV}$.

\section{CONCLUSIONS}

The influence of STM measurements on the decomposition of the oxide layer on $\mathrm{Si}(110)$ was analyzed. The STM tip etched the oxide surface and affected on the void shape. The decomposition reaction induced via STM should occur at defects, where the defect site acts as an electron trap and the $\mathrm{Si}-\mathrm{O}$ bonds were weak. From comparison to XPS estimation, the resultant activation energy reduction of the decomposition was estimated to be $0.4 \mathrm{eV}$ upon the influence of STM measurements.

\section{ACKNOWLEDGMENTS}

The XPS experiments were performed at the BL23SU of Spring-8 with the approval of the Japan Synchrotron Radiation Research Institute (JASRI) (Proposal Nos. 2016B3801 and 2016A3801).
[1] A. Ishizaka and Y. Shiraki, J. Electrochem. Soc. 133, 666 (1986).
[2] D. Jones and V. Palermo, Appl. Phys. Lett. 80, 673 (2002). 
[3] K. E. Johnson, P. K. Wu, M. Sander, and T. Engel, Surf. Sci. 290, 213 (1993).

[4] H. Watanabe, K. Fujita, and M. Ichikawa, Appl. Phys. Lett. 70, 1095 (1997).

[5] K. E. Johnson and T. Engel, Phys. Rev. Lett. 69, 339 (1992).

[6] G. W. Rubloff, J. Vac. Sci. Technol. A 8, 1857 (1990).

[7] R. Tromp, G. W. Rubloff, P. Balk, F. K. LeGoues, and E. J. van Loenen, Phys. Rev. Lett. 55, 2332 (1985).

[8] K. Fujita, H. Watanabe, and M. Ichikawa, Appl. Phys. Lett. 70, 2807 (1997).

[9] N. Miyata, H. Watanabe, and M. Ichikawa, Phys. Rev. Lett. 84, 1043 (2000).

[10] K. Fujita, H. Watanabe, and M. Ichikawa, Surf. Sci. 398, 134 (1998).

[11] Y. Wei, R. M. Wallace, and A. C. Seabaugh, Appl. Phys. Lett. 69, 1270 (1996).

[12] H. Hibino, M. Uematsu, and Y. Watanabe, J. Appl. Phys. 100, 113519 (2006).

[13] D. Fujita, J. Surf. Sci. Soc. Jpn. 36, 459 (2015) (in Japanese).

[14] H. Iwasaki, T. Yoshinobu, and K. Sudoh, Nanotechnology 14, R55 (2003).
[15] S. Fujita, S. Maruno, H. Watanabe, and M. Ichikawa, Appl. Phys. Lett. 69, 638 (1996).

[16] T. Satô, Y. Takeishi, H. Hara, and Y. Okamoto, Phys. Rev. B 4, 1950 (1971).

[17] W. Cheng, A. Teramoto, M. Hirayama, S. Sugawa, and T. Ohmi, Jpn. J. Appl. Phys. 45, 3110 (2006).

[18] M. Yano, Y. Uozumi, S. Yasuda, and H. Asaoka, Jpn. J. Appl. Phys. 57, 06HD04 (2018).

[19] H.-W. Fink, IBM J. Res. Dev. 30, 460, (1986).

[20] H. Watanabe, K. Fujita, and M. Ichikawa, Appl. Phys. Lett. 72, 1987 (1998).

[21] C. Detavernier, R. L. Van Meirhaeghe, F. Cardon, and K. Maex, Thin Solid Films 386, 19 (2001).

[22] Y. Yamamoto, T. Sueyoshi, T. Sato, and M. Iwatsuki, Surf. Sci. 466, 183 (2000).

[23] H. Togashi, Y. Takahashi, A. Kato, A. Konno, H. Asaoka, and M. Suemitsu, Jpn. J. Appl. Phys. 46, 3239 (2007).

[24] M. Suemitsu, A. Kato, H. Togashi, A. Konno, Y. Yamamoto, Y. Teraoka, A. Yoshigoe, Y. Narita, and Y. Enta, Jpn. J. Appl. Phys. 46, 1888 (2007). 